\title{
DESIGN, MODELING, AND CONTROL OF A WHEEL-LEGGED LOCOMOTION SYSTEM FOR THE ENVIRONMENTAL HYBRID ROBOT
}

\author{
Gustavo Freitas \\ Dept. of Electrical Eng. \\ COPPE/Federal University of Rio de Janeiro \\ Rio de Janeiro, Brazil \\ email: gfreitas@ coep.ufrj.br \\ Liu Hsu \\ Dept. of Electrical Eng. \\ COPPE/Federal University of Rio de Janeiro \\ Rio de Janeiro, Brazil \\ email: liu@coep.ufrj.br \\ Ney R. Salvi dos Reis \\ CENPES \\ Petrobras S.A. \\ Rio de Janeiro, Brazil \\ email: salvireis@petrobras.com.br
}

\begin{abstract}
Urucu, deep in the Amazon forest, is the largest in-land natural gas and oil producing site in Brazil. The oil and gas extracted in Urucu travel some $700 \mathrm{~km}$ in a pipeline through the jungle until they reach Manaus, where they are refined and consumed. Exploration of the site is conditioned on the Brazilian oil company Petrobras monitoring a $400 \mathrm{~km}$ section of the pipeline for any signs of water contamination. The Environmental Hybrid Robot (EHR) was conceived to automate this humongous monitoring task, currently executed in extremely small scale by scientists and locals on foot and small boats.

This paper describes the design, analysis, and testing of the EHR's second-generation locomotion mechanism, based on a two-DOF parallel mechanism. The new mechanism allows for independent control of wheel position and orientation relative to the terrain, thus significantly increasing the robot's mobility. Additionally, it allows for control of the vehicle's velocity-torque curve by adjusting the radius of curvature of the wheel at the point of contact with the soil. We present the forward and differential kinematic models of the new mechanism and, based on them, a differential kinematics-based position and orientation control method. We illustrate the methodology with experimental results.
\end{abstract}

\section{KEY WORDS}

Mobile Robots, Parallel Mechanisms, Hazardous Environments, Environmental Robotics.
Fernando Lizarralde

Dept. of Electrical Eng.

COPPE/Federal University of Rio de Janeiro

Rio de Janeiro, Brazil

email: fernando@coep.ufrj.br

Vitor Paranhos

Dept. of Electrical Eng.

COPPE/Federal University of Rio de Janeiro

Rio de Janeiro, Brazil

email: vitor.carneval@del.ufrj.br

Marcel Bergerman

Field Robotics Center - Robotics Institute

Carnegie Mellon University

Pittsburgh, USA

email: marcel@cmu.edu

\section{Introduction}

Large petrochemical operations in natural sanctuaries are always at risk of causing severe environmental damage. The Brazilian oil company Petrobras S.A. has a large oil and natural gas operation in Urucu, deep in the Amazon forest (Fig. 1). The oil and gas are transported in a $700 \mathrm{~km}$ pipeline to Manaus, where they are refined and consumed.

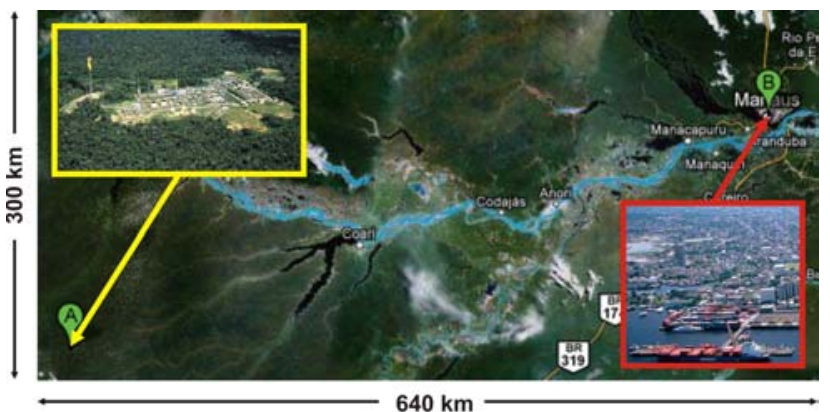

Figure 1. The Urucu-Manaus pipeline spans $700 \mathrm{~km}$ of pristine Amazon forest. The oil and gas extracted in Urucu (point A in the image) are consumed in Manaus (point B), a city with two million inhabitants.

To issue Petrobras a license to build the pipeline, the Brazilian government demanded that the company monitor water quality over a $400 \mathrm{~km}$ section of the pipeline connecting Coari to Manaus. Today this is performed by small groups of scientists and locals on foot and small boats (Fig. 2). Clearly, the large distances and huge areas involved, the varying types of surfaces (water, marshes, and dry land), not to mention the perils of tropical disease, en- 
counters with wildlife, etc., call for automated solutions that make the process safer and more efficient.

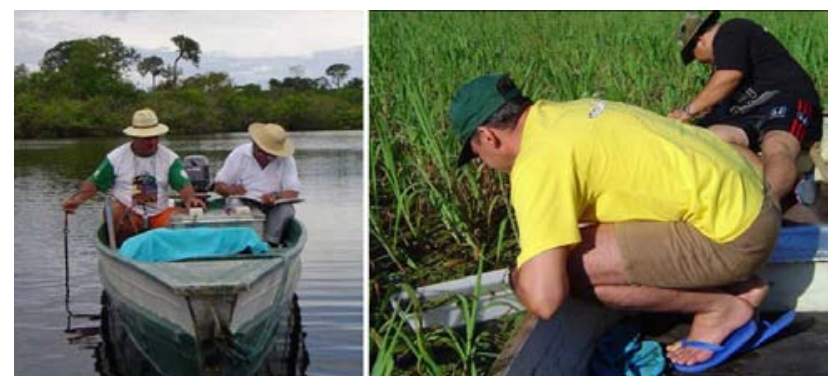

Figure 2. Typical monitoring activities in the Amazon forest near Coari. Researches measure water parameters using sampling sondes, and collect water samples with larvae of insects for posterior analysis. These insects transmit tropical diseases such as yellow fever, malaria, dengue, and leishmaniasis. Not always do people use personal protective equipment.

Petrobras is developing one such system. Named Environmental Hybrid Robot (EHR), it is a family of vehicles that can navigate on rivers and drive on dry land and marshes. The EHR family is currently composed of three vehicle models, each one about twice as large as the previous one (Fig. 3). The different models are employed according to specific mission characteristics, operation area, and sensing requirements. For example, the small vehicle is intended for short operations in forested regions where its large counterpart cannot maneuver, while the large one is used for long missions in open areas. All vehicles can be teleoperated, with the large one also capable of carrying a person to conduct the vehicle to regions of interest.

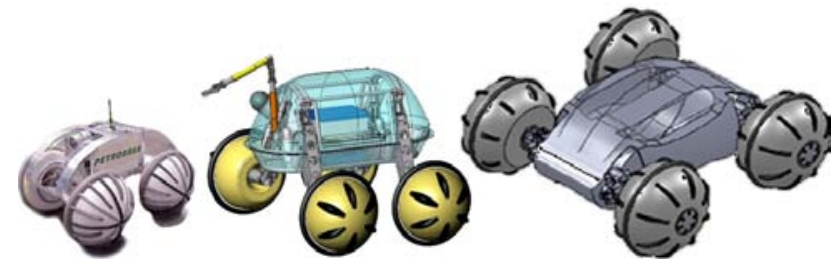

Figure 3. Environmental Hybrid Robot family. From left to right, the vehicles' wheel diameter is 300,600 , and $900 \mathrm{~mm}$.

To cope with the different surfaces it finds along the way, the EHR uses a wheel-legged design where each of four legs terminates in a large balloon-like profile. Controlling the position and orientation of the wheel is key to robust locomotion across the region, and is the subject of this paper.

This paper presents the new locomotion mechanism developed for the second generation EHR. The wheels are used for driving on land and propulsion on water; the internal articulation repositions the center of mass of the robot so it can deal with obstacles and adjust the contact forces against the terrain.
The new suspension is based on a planar parallel mechanism with two degrees-of-freedom (DOF), allowing for independent wheel position and orientation control. The parallel configuration was adopted because of its accuracy, repeatability and payload capacity. Even taking into account parallel structures' inherent disadvantages, such as limited workspace and complex forward kinematic and singularity analysis [14, 12, 1], in our case it was advantageous to use them because of their superior pose accuracy when compared to serial chain mechanisms $[9,10]$.

The two-DOF leg model is obtained based on the strategy presented in [14]. First, we obtain the mechanism's differential kinematic model from its kinematic constrains; then, taking into account the system's effective DOF, we apply a control strategy that relies on the model's null space to control a primary (position) and auxiliary (orientation) objectives. The controller is validated through actual experiments.

The paper is structured as follows. In Section 2 we present the new EHR locomotion system. In Sections 3 and 4 we present, respectively, the leg forward and differential kinematics. In Section 5 we present the kinematic controller, followed by experimental results in Section 6 . We conclude the paper in Section 7 with a discussion on future work to be performed.

\section{Environmental Hybrid Robot}

The Environmental Hybrid Robot is being developed to monitor the Amazon rain forest along the Coari-Manaus pipeline [8]. The EHR must operate in areas of difficult access and across a variety of surfaces (Fig. 4). It collects water, soil and vegetation samples for off-line analysis, as well as imagery from the river and forest. It is also capable of detecting combustible gases and measuring water parameters in situ, providing georeferenced data about the region. The EHR can also be employed in contingency operations, e.g., launching an oil absorption barrier on the river (Fig. 5).

In this section we first present the concepts behind the original design and the first-generation, one-DOF locomotion mechanism. We then present the current two-DOF system that is the basis of this paper's modeling and control results.

\subsection{Locomotion System Initial Design}

A robot's locomotion system must be designed according to its anticipated operating environment. The EHR operates in a region where the surface varies from open water to water with vegetation, marshes, swamps, firm ground with vegetation, and sandy ground. Perhaps even more crucially, it must be able to negotiate the transitions between them (Fig. 6). We adopted a wheel-legged architecture that accommodates these requirements and is able to carry the vehicle and sensors required for the monitoring task. Our de- 


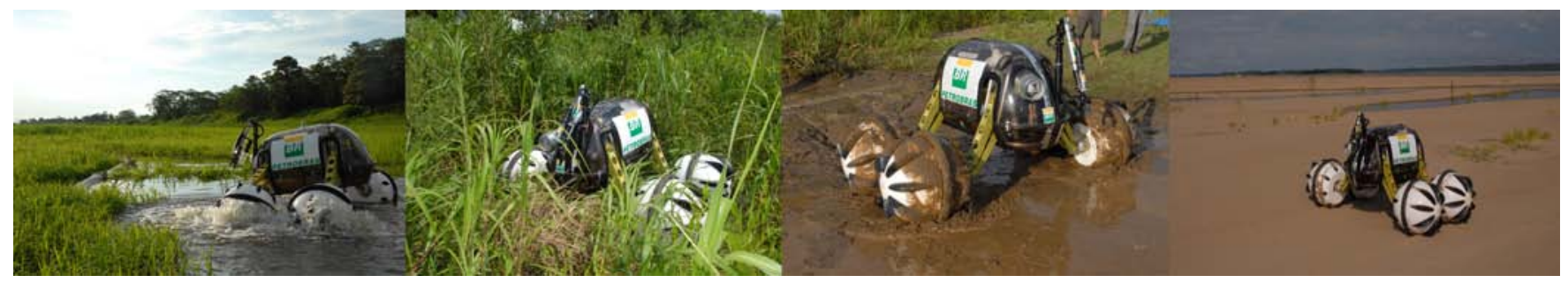

Figure 4. The types of surfaces the EHR must negotiate. From left to right: open water, water with vegetation, firm ground with vegetation/marshes, and sandy ground.

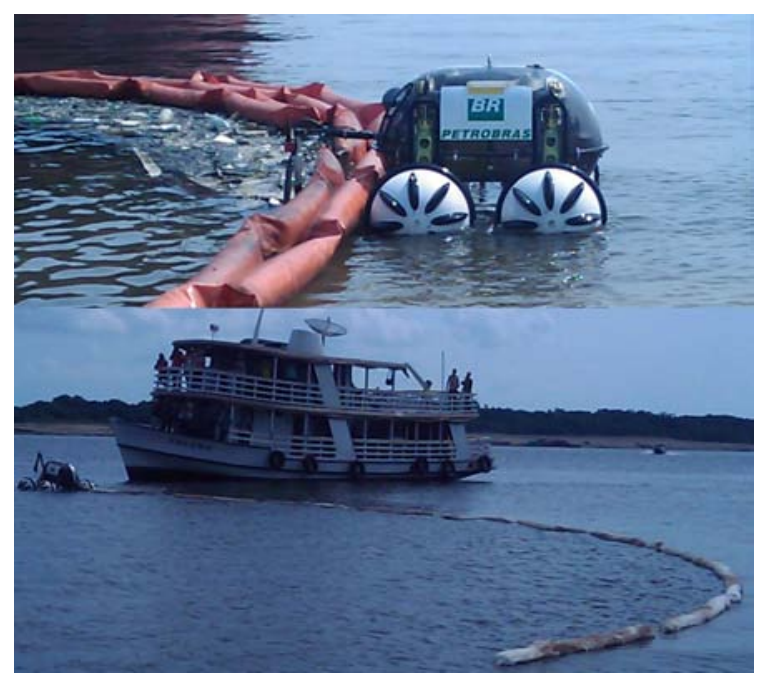

Figure 5. EHR missions: measuring water parameters and launching a $36 \mathrm{~m}$ oil absorption barrier on the Amazonas river.

sign incorporates large balloon-like wheel covers that provide floatation in water and traction in a variety of terrains.

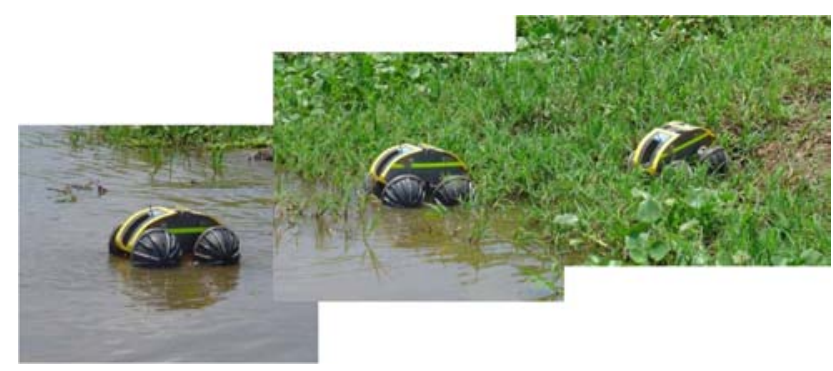

Figure 6. EHR progressing from water to marsh to land.

Each wheel is coupled to an independent suspension system, here referred to as the leg, composed of a spring and electric linear actuator. The suspension motors are attached to screws, constituting the active prismatic joints. The suspension's parallel mechanism is designed for structural rigidity - to allow the robot to overcome obstacles and wheel traction.
The first-generation suspension developed for the EHR is presented in Fig. 7. This mechanism has one DOF; therefore, when the coupled motor is actuated, both the position and the angle of the wheel relative to the terrain are affected.

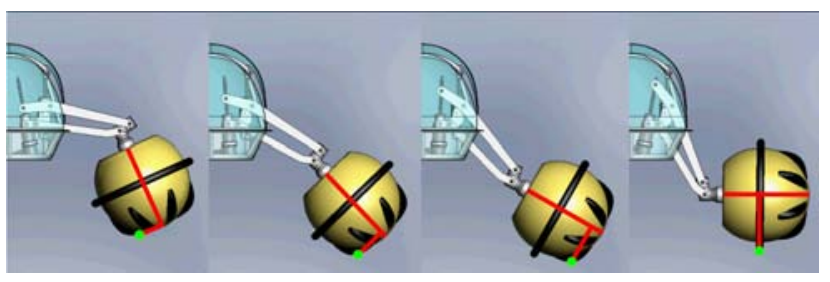

Figure 7. First-generation EHR with one-DOF locomotion mechanism. Wheel position and orientation change simultaneously because of the single actuation input.

By commanding the wheel's height, it is possible to modify the system's stability and force distribution among the legs $[6,5]$. The suspension angle also influences the contact point with the terrain and consequently the effective radius of the spherical wheels. Therefore, suspension reconfiguration affects the relationship between velocity and torque in each wheel.

While having proven effective during field tests in the Amazon, the first-generation EHR does not allow us to independently control either the vehicle's stability/force distribution or the relationship between velocity and torque. We then set out to redesign the suspension system, where control of the wheel height and angle are decoupled.

\subsection{New EHR Locomotion System}

The new EHR suspension is a two-DOF parallel mechanism. It has a larger workspace when compared to the previous version. This allows the robot to increase clearance during operations on water or place its body close to ground, which improves stability when moving on firm terrains. With the new suspension the robot is also capable of operating upside down, as shown in Fig. 8.

The robot pictured in Fig. 8 corresponds to the small version of the EHR family. It uses 20 " plastic wheels with solid tires that, during operation, are covered by a profile as 


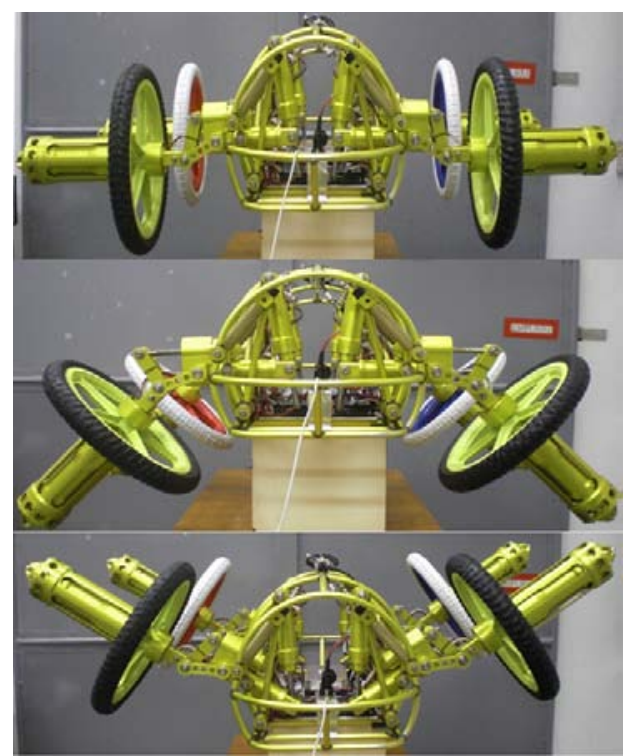

Figure 8. Second-generation EHR prototype with two-DOF locomotion mechanism. The picture does not show the balloon-like profiles that are mounted around the tires.

shown in Fig. 9. The robot weighs $35 \mathrm{~kg}$ and has a wheel base of $490 \mathrm{~mm}$ and track width of $710 \mathrm{~mm}$.

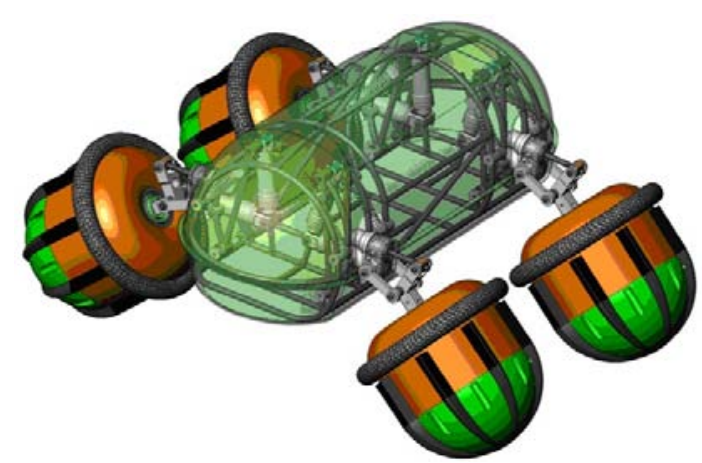

Figure 9. CAD model of the second-generation EHR with the profiles installed around the tires.

The robot is powered by NiMH batteries. It is equipped with a set of motors ( $70 \mathrm{~W}$ for each wheel and $10 \mathrm{~W}$ for each suspension) and drivers from Maxon Motors. The maximum speed on firm ground is of $1.5 \mathrm{~m} / \mathrm{s}$.

The system is teleoperated, and communication with the base computer is accomplished through $900 \mathrm{MHz}$ Ethernet radios. The embedded control algorithms are executed on a PC/104 board with a $366 \mathrm{MHz}$ processor running Linux. The system orientation is obtained by a MTI Xsens inertial unit.

\section{EHR Leg Forward Kinematics}

The first step in developing a controller for the EHR legs is the derivation of their forward and differential kinematic models. They are presented, respectively, in this and the next sections.

The leg geometry is presented in Fig. 10. It is a seven-bar linkage, where link 0 is fixed to the robot structure and link 6 corresponds to the wheel. The mechanism has two active prismatic joints and six passive revolute joints. Using Gluebler's formula [11] one can verify that this configuration results in two effective DOF's, corresponding to an underactuated planar mechanism with three open serial kinematic chains forming two closed chains [13]. We denote by $q_{a}$ the vector of active joint displacements $q_{a}=\left[d_{1}, d_{2}\right]^{T}$, and by $q_{p}$ the vector of passive joint displacements $q_{p}=\left[\theta_{1}, \theta_{2}, \theta_{3}, \theta_{4}, \theta_{5}, \theta_{6}\right]^{T}$.

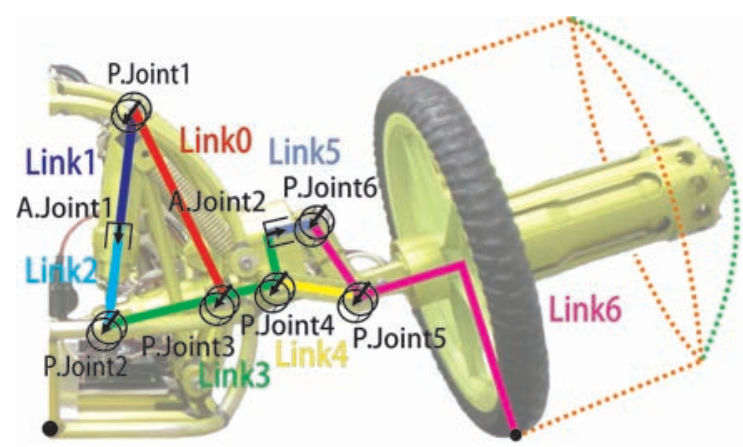

Figure 10. Each EHR leg is a seven-bar linkage mechanism with two active (A) and six passive (P) joints.

The forward kinematics is the geometric representation of the leg mechanism from base to a pre-selected terminal point. We adopt as terminal point that where the wheel contacts the terrain, which is also the lowest point on the wheel (Fig. 10). (In our future work we will extend the analysis to consider the balloon-like wheel cover, where the lowest point on the wheel may not coincide with the contact point with the terrain.) The coordinate frame attached to the $i$-th link is denoted $\left\{x_{i}, y_{i}\right\}$ (see Fig. 11). The wheel-terrain contact position is defined by frame $\left\{x_{w}, y_{w}\right\} . \vec{p}_{i j} \in \mathbb{R}^{3}$ is the vector from the origin of frame $i$ to the origin of frame $j$, written with respect to the base frame (frame 0 ). The kinematic parameters are shown in Table 1.

With the definitions above, the forward kinematics can be written in terms of the three kinematic chains as 


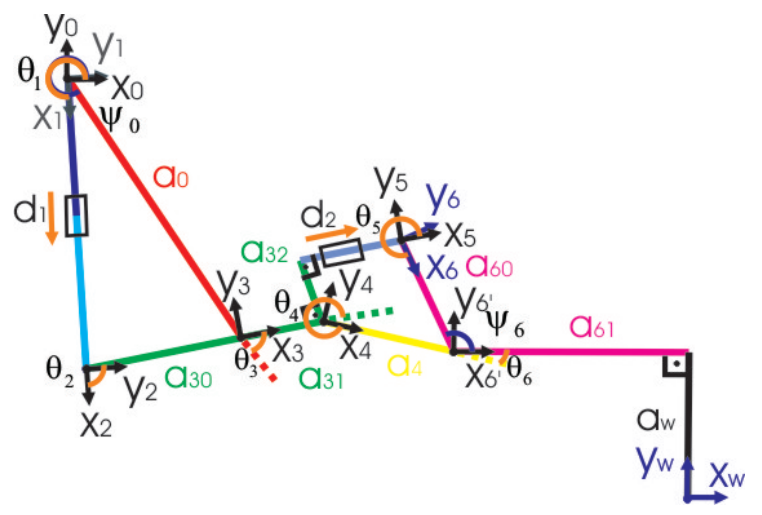

Figure 11. EHR leg's coordinate frames.

Table 1. EHR leg's kinematic parameters.

\begin{tabular}{|c|c|c|c|}
\hline Link \# & Parameter & Value & Unit \\
\hline 0 & $a_{0}$ & 185 & $\mathrm{~mm}$ \\
& $\psi_{0}$ & 117.0 & $\mathrm{deg}$ \\
\hline 3 & $a_{30}$ & 80 & $\mathrm{~mm}$ \\
& $a_{31}$ & 40 & $\mathrm{~mm}$ \\
& $a_{32}$ & 40 & $\mathrm{~mm}$ \\
\hline 4 & $a_{4}$ & 70 & $\mathrm{~mm}$ \\
\hline 6 & $a_{60}$ & 75 & $\mathrm{~mm}$ \\
& $a_{61}$ & 115 & $\mathrm{~mm}$ \\
& $a_{w}$ & 275 & $\mathrm{~mm}$ \\
& $\psi_{6}$ & 112.0 & $\mathrm{deg}$ \\
\hline
\end{tabular}

the following structure equation [11]:

$$
\begin{aligned}
\vec{p}_{0 w} & =\underbrace{\vec{p}_{02}+\vec{p}_{24}+\vec{p}_{46^{\prime}}+\vec{p}_{6^{\prime} w}}_{\text {chain } 1}=\underbrace{\vec{p}_{02}+\vec{p}_{24}+\vec{p}_{46}+\vec{p}_{6 w}}_{\text {chain } 2} \\
& =\underbrace{\vec{p}_{03}+\vec{p}_{34}+\vec{p}_{46^{\prime}}+\vec{p}_{6^{\prime} w}}_{\text {chain } 3} \\
\theta_{0 w} & =\underbrace{\theta_{1}+\theta_{2}+\theta_{4}+\theta_{6}}_{\text {chain } 1}=\underbrace{\theta_{1}+\theta_{2}+2 \pi+\theta_{5}+\pi-\psi_{6}}_{\text {chain } 2} \\
& =\underbrace{\psi_{0}+\theta_{3}+\theta_{4}+\theta_{6}}_{\text {chain } 3}
\end{aligned}
$$

where $\theta_{0 w}$ represents the orientation of the wheel frame with respect to the base frame.

Equations (1)-(2) represent the constraints that the passive joints must obey given the underactuated nature of the leg mechanism. For each pair $\left[d_{1}, d_{2}\right]$, these equations can be solved to yield the angles of the passive joints. given by:

From Equation (1), the forward kinematic model is

$$
\begin{aligned}
\vec{p}_{0 w}= & \vec{p}_{02}+\vec{p}_{24}+\vec{p}_{46^{\prime}}+\vec{p}_{6^{\prime} w} \\
= & d_{2} R_{01}\left(\theta_{1}\right) x+\left(a_{30}+a_{31}\right) R_{01}\left(\theta_{1}\right) R_{23}\left(\theta_{2}\right) x \\
& +a_{4} R_{01}\left(\theta_{1}\right) R_{23}\left(\theta_{2}\right) R_{34}\left(\theta_{4}\right) x \\
& +a_{61} R_{01}\left(\theta_{1}\right) R_{23}\left(\theta_{2}\right) R_{34}\left(\theta_{4}\right) R_{46^{\prime}}\left(\theta_{6}\right) x \\
& -a_{w} R_{01}\left(\theta_{1}\right) R_{23}\left(\theta_{2}\right) R_{34}\left(\theta_{4}\right) R_{46^{\prime}}\left(\theta_{6}\right) y
\end{aligned}
$$

where $a_{30}, a_{31}, a_{4}, a_{61}, a_{w}$ are given in Table $1, x=$

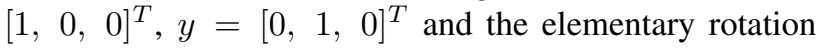
matrix $R_{i j}\left(\theta_{i}\right) \in S O(3)$ is the orientation of frame $j$ with respect to frame $i$, given by:

$$
R_{i j}\left(\theta_{i}\right)=\left[\begin{array}{ccc}
\cos \left(\theta_{i}\right) & -\sin \left(\theta_{i}\right) & 0 \\
\sin \left(\theta_{i}\right) & \cos \left(\theta_{i}\right) & 0 \\
0 & 0 & 1
\end{array}\right]
$$

Due to the parallel mechanism structure constraints, it is not trivial to obtain an analytical solution for the forward kinematics in terms of $q_{a}$, i.e., $\vec{p}_{0 w}=f_{1}\left(d_{1}, d_{2}\right), \theta_{0 w}=$ $f_{2}\left(d_{1}, d_{2}\right)$.

The passive joint vector $q_{p} \in \mathbb{R}^{6}$ can be obtained in terms of the active joints $d_{1}, d_{2}$ through algebraic identities and the geometry of the mechanism. Another possible way to determine $q_{p}$ is to reduce the forward kinematic problem into appropriate subproblems whose solutions are known [11]. The passive joints $q_{p}$ can then be calculated through Paden-Kahan subproblems 1 and 3 [11, pag. 99103], which are geometrically meaningful and numerically stable.

Once $q_{p}$ is known, it is possible to obtain the contact point position $\vec{p}_{0 w}$ via Equation (3) and its orientation $\theta_{0 w}$ via Equation (2). Figure 12 illustrates the configuration of one leg for $d_{1} \in[170,220] \mathrm{mm}$ and $d_{2} \in[45,105] \mathrm{mm}$. The mechanism dextrous workspace in terms of $p_{0 w}$ and $\theta_{0 w}$ is presented in Fig. 13.

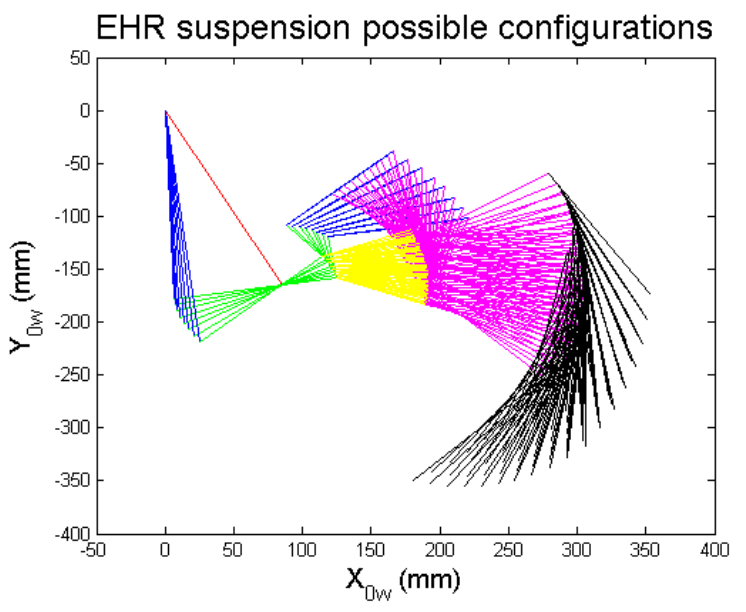

Figure 12. Configurations of the EHR leg in terms of $q_{a}$.

\section{EHR Leg Differential Kinematics}

To find the relationship between the velocity of the wheelterrain contact point and the velocity of the joints, we differentiate Equation (1), thus obtaining a Jacobian matrix 


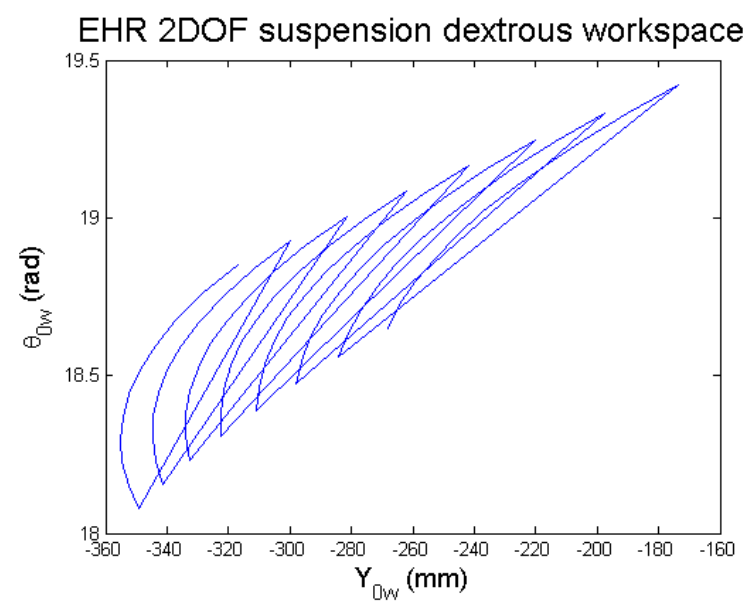

Figure 13. Dextrous workspace of one EHR leg.

for each open serial chain:

$$
v_{w}=S J_{1}\left[\begin{array}{c}
\dot{\theta}_{1} \\
\dot{d}_{1} \\
\dot{\theta}_{2} \\
\dot{\theta}_{4} \\
\dot{\theta}_{6}
\end{array}\right]=S J_{2}\left[\begin{array}{c}
\dot{\theta}_{1} \\
\dot{d}_{1} \\
\dot{\theta}_{2} \\
\dot{d}_{2} \\
\dot{\theta}_{5}
\end{array}\right]=S J_{3}\left[\begin{array}{c}
\dot{\theta}_{3} \\
\dot{\theta}_{4} \\
\dot{\theta}_{6}
\end{array}\right]
$$

where $v_{w}=\left[\dot{p}_{0 w_{x}}, \dot{p}_{0 w_{y}}, \dot{\theta}_{0 w}\right]^{T} \in \mathbb{R}^{3}$ is the vector that combines the planar linear velocity in the $x_{0}, y_{0}$ axes and the angular velocity $\dot{\theta}_{0 w} . S \in \mathbb{R}^{3 \times 6}$ is the following selection matrix:

$$
S=\left[\begin{array}{llllll}
1 & 0 & 0 & 0 & 0 & 0 \\
0 & 1 & 0 & 0 & 0 & 0 \\
0 & 0 & 0 & 0 & 0 & 1
\end{array}\right]
$$

The Jacobian matrices are given by

$$
\begin{aligned}
& J_{1}=\left[\begin{array}{ccccc}
z \times \vec{p}_{0 w} & R_{01} x & z \times \vec{p}_{2 w} & z \times \vec{p}_{4 w} & z \times \vec{p}_{6^{\prime} w} \\
z & 0 & z & z & z
\end{array}\right] \\
& J_{2}=\left[\begin{array}{ccccc}
z \times \vec{p}_{0 w} & R_{01} x & z \times \vec{p}_{2 w} & R_{01} R_{23} x & z \times \vec{p}_{6 w} \\
z & 0 & z & 0 & z
\end{array}\right] \\
& J_{3}=\left[\begin{array}{ccc}
z \times \vec{p}_{3 w} & z \times \vec{p}_{4 w} & z \times \vec{p}_{6^{\prime} w} \\
z & z & z
\end{array}\right]
\end{aligned}
$$

where $z=[0,0,1]^{T}, \vec{p}_{0 w}$ is given by Equation (3), and

$$
\begin{aligned}
\vec{p}_{2 w}= & \left(a_{30}+a_{31}\right) R_{01}\left(\theta_{1}\right) R_{23}\left(\theta_{2}\right) x+\vec{p}_{4 w} \\
\vec{p}_{3 w}= & a_{31} R\left(\psi_{0}\right) R_{03}\left(\theta_{3}\right) x+\vec{p}_{4 w} \\
\vec{p}_{4 w}= & a_{4} R_{01}\left(\theta_{1}\right) R_{23}\left(\theta_{2}\right) R_{34}\left(\theta_{4}\right) x+\vec{p}_{6^{\prime} w} \\
\vec{p}_{6 w}= & a_{60} R_{01}\left(\theta_{1}\right) R_{23}\left(\theta_{2}\right) R_{36}\left(\theta_{5}\right) p_{6 w} \\
p_{6 w}= & {\left[a_{60} \cos \psi_{6}+a_{61} ;-a_{60} \sin \psi_{6}-a_{w} ; 0\right]^{T} } \\
\vec{p}_{6^{\prime} w}= & a_{61} R_{01}\left(\theta_{1}\right) R_{23}\left(\theta_{2}\right) R_{34}\left(\theta_{4}\right) R_{46^{\prime}}\left(\theta_{6}\right) x \\
& -a_{w} R_{01}\left(\theta_{1}\right) R_{23}\left(\theta_{2}\right) R_{34}\left(\theta_{4}\right) R_{46^{\prime}}\left(\theta_{6}\right) y
\end{aligned}
$$

Stacking the three individual Jacobians for each chain we find the extended Jacobian $J_{e x t}$ :

$$
\underbrace{\left[\begin{array}{ccc}
S J_{1} & 0 & 0 \\
0 & S J_{2} & 0 \\
0 & 0 & S J_{3}
\end{array}\right]}_{J_{e x t}} \dot{q}_{e x t}=\underbrace{\left[\begin{array}{c}
I \\
I \\
I
\end{array}\right]}_{A} v_{w}
$$

or, equivalently:

$$
J_{e x t} \dot{q}_{e x t}=A v_{w}
$$

where matrix $A \in \mathbb{R}^{9 \times 3}$ is always full column rank, $J_{\text {ext }} \in$ $\mathbb{R}^{9 \times 13}$ and $\dot{q}_{e x t}=\left[\dot{\theta}_{1}, \dot{d}_{1}, \dot{\theta}_{2}, \dot{\theta}_{4}, \dot{\theta}_{6}, \dot{\theta}_{1}, \dot{d}_{1}, \dot{\theta}_{2}, \dot{d}_{2}, \dot{\theta}_{5}, \dot{\theta}_{3}\right.$, $\left.\dot{\theta}_{4}, \dot{\theta}_{6}\right]^{T}$.

Note that joints $\dot{\theta}_{1}, \dot{d}_{1}, \dot{\theta}_{2}, \dot{\theta}_{4}, \dot{\theta}_{6}$ are duplicated in Equation (12), and, therefore, $J_{\text {ext }}$ has more columns than necessary. Defining $q_{e x t}=M q$, with

$$
M=\left[\begin{array}{ccc}
I^{3} & 0^{3 \times 2} & 0^{3 \times 3} \\
0^{2 \times 3} & I^{2} & 0^{2 \times 3} \\
I^{3} & 0^{3 \times 2} & 0^{3 \times 3} \\
0^{3 \times 3} & 0^{3 \times 2} & I^{3} \\
0^{2 \times 3} & I^{2} & 0^{2 \times 3}
\end{array}\right],
$$

where $I^{i}$ is the identity matrix of order $i$ and $0^{i \times j}$ is the null matrix of order $i \times j$, Equation (12) can be rewritten as:

$$
J \dot{q}=A v_{w}
$$

with $J \in \mathbb{R}^{9 \times 8}=J_{\text {ext }} M$ and $\dot{q}=\left[\dot{\theta}_{1}, \dot{d}_{1}, \dot{\theta}_{2}, \dot{\theta}_{4}, \dot{\theta}_{6}, \dot{d}_{2}\right.$, $\left.\dot{\theta}_{5}, \dot{\theta}_{3}\right]^{T}$.

Let $A^{\dagger} \in \mathbb{R}^{3 \times 9}$ be the pseudo-inverse of $A$ such that $A^{\dagger} A=I$, and $\widetilde{A} \in \mathbb{R}^{6 \times 9}$ be its annihilator such that $\widetilde{A} A=$ 0 . Then the differential kinematic relationship (14) can be equivalently written as $[12,14]$ :

$$
v_{w}=J_{t} \dot{q}, \quad J_{c} \dot{q}=0
$$

where $J_{t}=A^{\dagger} J$ and $J_{c}=\tilde{A} J$.

Partitioning $J_{t} \in \mathbb{R}^{3 \times 8}$ and $J_{c} \in \mathbb{R}^{6 \times 8}$ into components related to the active and passive joints, i.e.,

$$
J_{t}=\left[J_{t a} ; J_{t p}\right], \quad J_{c}=\left[J_{c a} ; J_{c p}\right]
$$

Equation (15) can be rewritten as:

$$
v_{w}=J_{t a} \dot{q}_{a}+J_{t p} \dot{q}_{p}, \quad J_{c a} \dot{q}_{a}+J_{c p} \dot{q}_{p}=0
$$

where $J_{t a} \in \mathbb{R}^{3 \times 2}, J_{t p} \in \mathbb{R}^{3 \times 6}, J_{c a} \in \mathbb{R}^{6 \times 2}$ and $J_{c p} \in \mathbb{R}^{6 \times 6}$.

For the EHR leg, $J_{c p}$ is always full rank inside the work envelope. Therefore, the passive joints velocities can be written in terms of the active joint velocities as:

$$
\dot{q}_{p}=-J_{c p}^{-1} J_{c a} \dot{q}_{a}
$$

Finally, the wheel velocity $v_{w}$ is given in terms of the active joints velocity $\dot{q}_{a}$ by:

$$
v_{w}=\underbrace{\left(J_{t_{a}}-J_{t_{p}} J_{c_{p}}^{-1} J_{c_{a}}\right)}_{J_{p}} \dot{q}_{a}
$$

where $J_{p} \in \mathbb{R}^{3 \times 2}$ is the effective leg Jacobian. 


\section{EHR Leg Kinematic Control}

The two fundamental quantities to be controlled in the EHR locomotion system are: (1) the vertical distance between the wheel-terrain contact point and the suspension frame, $p_{0 w_{y}}$, and (2) the wheel orientation $\theta_{0 w}$. The former stems from the fact that $p_{0 w_{y}}$ directly determines the robot's orientation, force distribution among legs, and tip-over stability $[5,4]$; and the latter from the fact that it directly determines the velocity-torque relationship by changing the effective wheel radius. In other words, the control objectives are to:

1. minimize the error between a reference vertical distance $p_{0 w_{y}}^{*}$ between the wheel-terrain contact point and the suspension frame and its actual value $p_{0 w_{y}}$ :

$$
e_{h}=p_{0 w_{y}}^{*}-p_{0 w_{y}}
$$

2. minimize the error between a reference wheel orientation $\theta_{0 w}^{*}$ and its actual value $\theta_{0 w}$ :

$$
e_{o}=\theta_{0 w}^{*}-\theta_{0 w}
$$

As discussed in Section 3, the EHR has two effective DOF; therefore it is theoretically possible to independently control $p_{0 w_{y}}$ and $\theta_{0 w}$ using a traditional multi-input-multioutput (MIMO) PID controller. For that, we would first partition the differential kinematic model (19) as:

$$
\left[\begin{array}{c}
\dot{p}_{0 w_{y}} \\
\dot{\theta}_{0 w}
\end{array}\right]=\bar{J}_{p} \dot{q}_{a} ; \quad \bar{J}_{p}=\left[\begin{array}{c}
J_{p_{y}} \\
J_{\theta}
\end{array}\right]
$$

where $J_{p_{y}}, J_{\theta} \in \mathbb{R}^{1 \times 2}$. Then, we would invert Equation (22) to obtain $\dot{q}_{a}=\bar{J}_{p}^{-1} v$, where $v$ is a PID-type auxiliary control input.

For the EHR, however, a standard MIMO controller cannot be used because of the geometric limitations of the mechanism. To see that, consider Fig. 13, which shows that not all possible orientations $\theta_{0 w}$ are achievable for a given $p_{0 w_{y}}$. For example, for $p_{0 w_{y}}<-320 \mathrm{~mm}$, it is no longer possible to orient the wheel perpendicular to the terrain, although it is possible to control $p_{0 w_{y}}$. Therefore, we choose to treat the EHR leg as a redundant mechanism with two inputs $u(t)=\dot{q}_{a}=\left[\dot{d}_{1}, \dot{d}_{2}\right]$ and one output $p_{0 w_{y}}$, and adopt as the primary control objective to make $e_{h} \rightarrow 0$. We then use the null-space control formulation $[13,3]$ to minimize the orientation error as a secondary control objective, i.e., to make $e_{o} \rightarrow 0$ within the leg's geometric limitations.

To design the controller we invert the first line of (22):

$$
\dot{q}_{a}=J_{p_{y}}^{\dagger} v+\underbrace{\left(I-J_{p_{y}}^{\dagger} J_{p_{y}}\right)}_{\mathcal{P}} \dot{q}_{a_{0}}
$$

where $\operatorname{col}(\mathcal{P})$ spans the null space of $J_{p_{y}}$, and $\dot{q}_{a_{0}}$ is an arbitrary vector of active joints velocities that can be selected to achieve a secondary control objective. Because the second term on the right-hand side of (23) operates on the null space of $J_{p_{y}}$, it does not influence control of the vertical distance $p_{0 w_{y}}$.

In the absence of modeling errors or external disturbances, and using the fact that $J_{p_{y}}\left(I-J_{p_{y}}^{\dagger} J_{p_{y}}\right)=0$, a simple proportional law such as

$$
u=J_{p_{y}}^{\dagger} K_{h} e_{h}+\left(I-J_{p_{y}}^{\dagger} J_{p_{y}}\right) \bar{u}
$$

where $\bar{u}$ is an auxiliary control signal, forces the error dynamic to follow $\dot{e}_{h}+K_{h} e_{h}=0$. Therefore, for a positivedefinite $K_{h}$ and nonsingular $J_{p_{y}}, \lim _{t \rightarrow \infty} e_{h}(t)=0$.

The auxiliary control signal $\bar{u}$ is of the form

$$
\bar{u}=\bar{K}\left(\frac{\partial f\left(q_{a}\right)}{\partial q_{a}}\right)^{T}
$$

where $\bar{K}>0$ is a gain factor and $f\left(q_{a}\right)$ is a continuous, differentiable and convex objective function in terms of $q_{a}$. Here we select $f\left(q_{a}\right)$ to cancel the orientation error, i.e., $f\left(q_{a}\right)=e_{o}^{2}$. The auxiliary control is then given by:

$$
\bar{u}=K_{o} e_{o} \dot{e}_{o}=K_{o} J_{\theta}^{T} e_{o}
$$

where $K_{o}$ is a positive-definite orientation gain matrix.

In summary, the active joints velocity $\dot{q}_{a}$ that minimizes the errors (20) and (21) is computed as:

$$
u=\dot{q}_{a}=J_{p_{y}}^{\dagger} K_{h} e_{h}+\left(I-J_{p_{y}}^{\dagger} J_{p_{y}}\right) K_{o} J_{\theta}^{T} e_{o}
$$

\section{Implementation and Experimental Results}

The control approach (27) was implemented on the secondgeneration EHR.

The control algorithm is executed onboard the robot on a PC/104 single board computer featuring a low-power, $366 \mathrm{MHz}$ AMD Geode GX500 processor running Linux (Debian 5.0.1, Kernel 2.6.34). To achieve real time code execution, we use Xenomai 2.5.3 as a layer on top of Linux [7]. Xenomai uses the Adaptive Domain Environment for Operating Systems (ADEOS) to implement the hardware interrupts that guarantee soft real-time operation. The implementation described here uses Xenomai's native API.

The EHR software is implemented in $\mathrm{C} / \mathrm{C}++$. The user-space application spawns the following real-time task that executes the control algorithm:

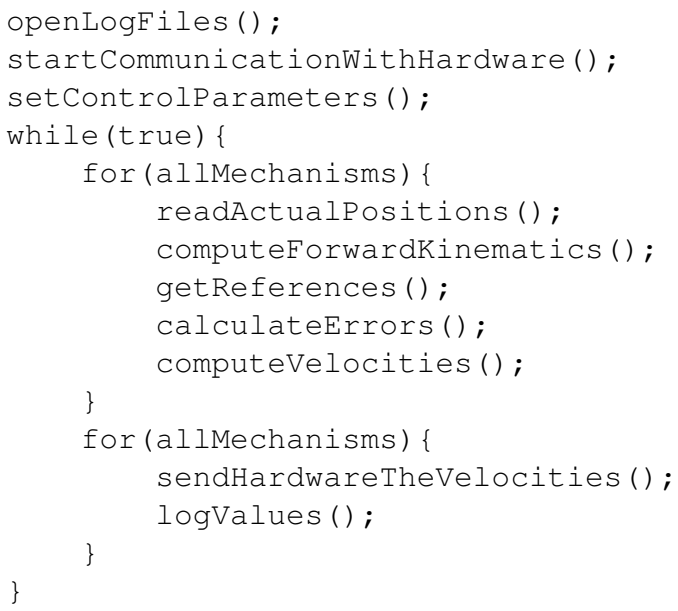


Among the several custom functions that comprise the code, the following two are particularly relevant:

- forwardKinematics(): Calculates the leg forward kinematics $\vec{p}_{0 w}, \theta_{0 w}$ from the active joint position vector $q_{a}$.

- suspensionControl(): Calculates the control command $u=\dot{q}_{a}$ from the joint position vectors $q_{a}, q_{p}$ and closed-loop position and orientation errors $e_{h}, e_{o}$.

The function forwardKinematics is obtained with Matlab's ccode command. The mathematical expression for the forward leg kinematics is declared as a symbolic expression in Matlab, and ccode is called to generate the $\mathrm{C}$ code implementing the expression. Because ccode does not always generate computationally-efficient code, we hand-customized the code to reduce computation speedby storing in memory those variables that are calculated frequently and reusing them during code execution. This manual optimization step resulted in code that runs $80 \%$ faster (see Table 2).

The suspensionControl function is also obtained via ccode and manual optimization. The matrix inversion $J_{c p}^{-1}$ is computed using the open source OpenCV computer vision library. OpenCV provides functions for inverting matrices using singular value decomposition (SVD) as well as Gaussian elimination with optimal pivot element selection (LU). The computational effort for both the original and optimized codes using each of the matrix inversion methods is shown in Table 3 . The optimized code with LU inversion runs $38 \%$ faster than its original counterpart.

Table 2. forwardKinematics computing mean time $\mathrm{E}[\mathrm{t}]$ and associated standard deviation $\sigma$.

\begin{tabular}{|l|c|c|}
\hline Algorithm & $\mathbf{E}[\mathbf{t}]$ & $\sigma$ \\
\hline Non-Optimized forwardKinematics & $165 \mu \mathrm{s}$ & $74.7 \mu \mathrm{s}$ \\
\hline Optimized forwardKinematics & $35 \mu \mathrm{s}$ & $6.5 \mu \mathrm{s}$ \\
\hline
\end{tabular}

Table 3. suspensionControl computing mean time $\mathrm{E}[\mathrm{t}]$ and associated standard deviation $\sigma$.

\begin{tabular}{|l|c|c|}
\hline Algorithm & $\mathbf{E}[\mathbf{t}]$ & $\sigma$ \\
\hline Non-Optimized with LU inversion & $353 \mu \mathrm{s}$ & $99.8 \mu \mathrm{s}$ \\
\hline Non-Optimized with SVD inversion & $1,074 \mu \mathrm{s}$ & $158.9 \mu \mathrm{s}$ \\
\hline Optimized with LU inversion & $219 \mu \mathrm{s}$ & $65.0 \mu \mathrm{s}$ \\
\hline Optimized with SVD inversion & $942 \mu \mathrm{s}$ & $175.6 \mu \mathrm{s}$ \\
\hline
\end{tabular}

In the experiments reported here the reference wheel vertical position $p_{0 w_{y}}^{*}$ is a series of step signals, and the reference orientation is such that the wheel is perpendicular to the ground. The results are presented in Fig. 14. It can be seen that the primary objective-control of the vertical position of the wheel-terrain contact point-is accomplished, with $e_{h} \rightarrow 0$ with a time constant of about $3 \mathrm{~s}$.

Likewise, the secondary control objective is also achieved, with the orientation error $e_{o}$ tending to zero at every change in reference position. Because of the reduced priority placed on orientation control, the time constant is longer-on the order of $20 \mathrm{~s}$. Note that the orientation error is not canceled when the active joint $d_{1}$ reaches its minimum position at $t=[140,160] \mathrm{s}$. This, however, is a physical limitation of the leg mechanism and not an intrinsic limitation of the controller. More importantly, even at these configurations the null-space control methodology still allows for full control of the vertical distance $p_{0 w_{y}}$, since the orientation control acts on the null space of $J_{p_{y}}$ and therefore does not interfere with control of $p_{0 w_{y}}$.
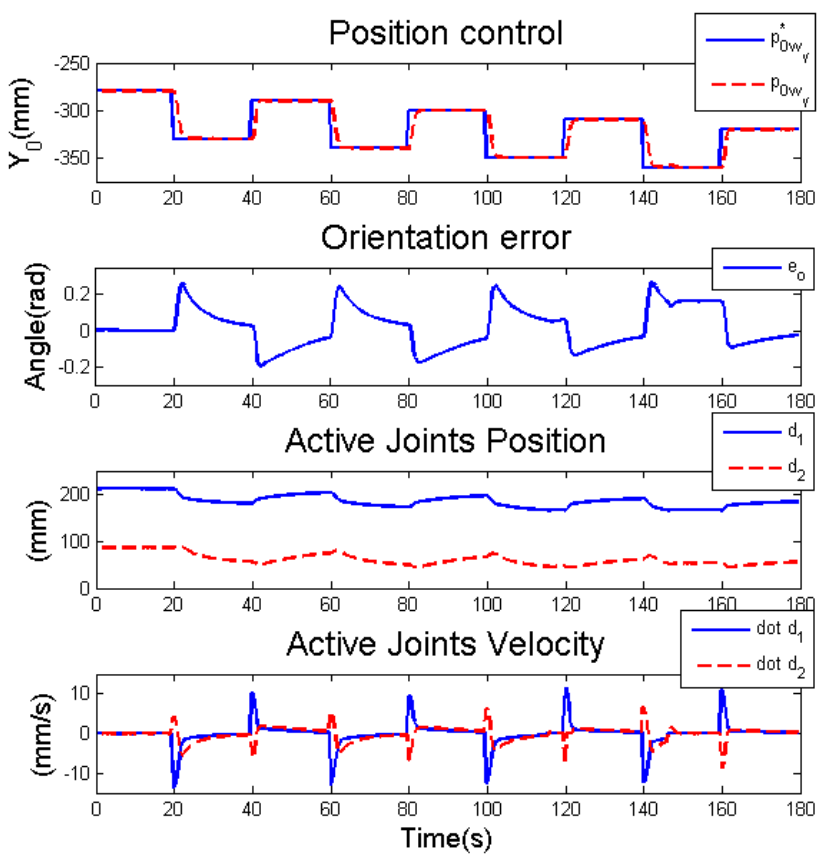

Figure 14. Implementation of the control method to the EHR leg. From top to bottom: position $p_{0 w_{y}}$, orientation $\theta_{0 w}$, active joint displacements $d_{1}, d_{2}$, and active joint velocities $\dot{d}_{1}, \dot{d}_{2}$.

\section{Conclusion}

This paper presented the new two-DOF wheel-legged locomotion system of the second-generation Environmental Hybrid Robot. The EHR is a family of vehicles designed and built by the Brazilian oil company Petrobras to monitor water quality around the $400 \mathrm{~km}$ long Coari-Manaus pipeline that runs through the heart of the Amazon forest. The paper detailed the leg's kinematic modeling and control of the wheel's position and orientation.

Because of the geometric nature of the leg mechanism, a standard MIMO controller would not be effective. 
Instead, we use a null-space control formulation that minimizes the error in the vertical distance between the wheelterrain contact point and the suspension frame as a primary control objective, while minimizing the error in the wheel orientation as a secondary objective. We demonstrated the controller efficacy through experimental results. Figure 15 shows the new robot during preliminary field tests in Rio de Janeiro.

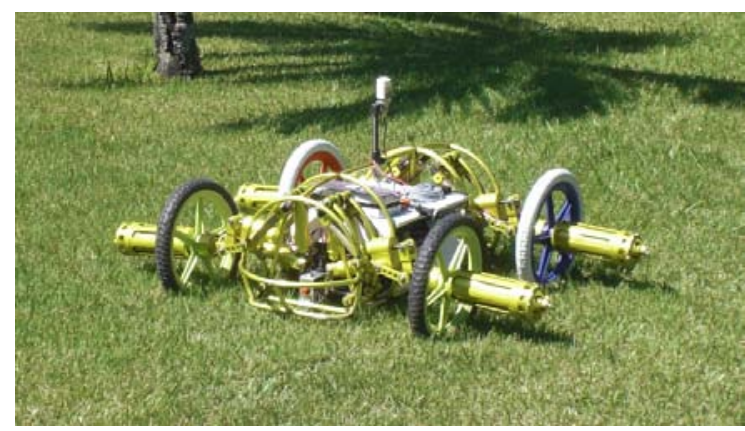

Figure 15. Second-generation EHR prototype during field tests.

Our next steps include: (1) implement and test a mobility methodology that optimizes vehicle stability and force distribution by actively controlling each leg's height and angle [5, 4]; (2) develop and test methods to adjust wheel-surface contact angle-and consequently the velocity-torque relationship-to control robot speed while minimizing power consumption and avoiding motor saturation [2]; and (3) continue deployment in the Amazon region. Based on field results we will determine the need to implement a more complex control method that explicitly takes into account modeling uncertainties and external disturbances.

\section{Acknowledgments}

The authors would like to thank all the Petrobras S.A. Robotics Laboratory team. This work was partially supported by FAPERJ, CNPq and Petrobras S.A.

\section{References}

[1] H. Abdellatif and B. Heimann, "Advanced modelbased control of a 6-dof hexapod robot: A case study," Mechatronics, IEEE/ASME Transactions on, vol. 15, no. 2, pp. 269-279, 2010.

[2] A. Barral, A. Santos, and M. A. Meggiolaro, "Traction control of all-wheel-drive independent suspension mobile robots in 2D rough terrain," in Brazilian Society of Mechanical Sciences and Engineering, Symposium Series in Mechatronics v.3, Rio de Janeiro, 2008, pp. 256-265.

[3] S. Chiaverini, G. Oriolo, and I. D. Walker, "Kinematically redundant manipulators," in Springer Handbook of Robotics, 1st ed., B. Siciliano and O. Khatib, Eds. Springer-Verlag Ltd., 2008, pp. 245-268.

[4] G. Freitas, G. Gleizer, F. Lizarralde, and L. Hsu, "Multi-objective optimization for kinematic reconfiguration of mobile robots," in Proc. of the 6th annual IEEE Conf. on Automation Science and Eng., Toronto, August 2010, pp. 686-691.

[5] G. Freitas, G. Gleizer, F. Lizarralde, L. Hsu, and N. R. S. dos Reis, "Kinematic reconfigurability control for an environmental mobile robot operating in the amazon rain forest," Journal of Field Robotics, vol. 27, no. 2, pp. 197-216, March-April 2010.

[6] G. Freitas, F. Lizarralde, L. Hsu, and N. R. S. dos Reis, "Kinematic reconfigurability of mobile robot on irregular terrains," in Proceedings of IEEE International Conference on Robotics \& Automation, Kobe, May 2009, pp. 1340-1345.

[7] P. Gerum, "Xenomai: Implementing a RTOS emulation framework on GNU/Linux," www.xenomai.org/documentation/branches/v2.3.x/ pdf/xenomai.pdf, 2004.

[8] E. Guizzo, "Dream jobs 2008 - Ney Robinson Salvi dos Reis: Into the wild," IEEE Spectrum Magazine, vol. 45, no. 2, pp. 33-34, February 2008.

[9] J.-P. Merlet, "Parallel manipulators: state of the art and perspectives," Advanced Robotics, vol. 8, no. 6, pp. 589-596, 1993.

[10] J.-P. Merlet and C. Gosselin, "Parallel mechanisms and robots," in Springer Handbook of Robotics, 1st ed., B. Siciliano and O. Khatib, Eds. SpringerVerlag Ltd., 2008, pp. 269-285.

[11] R. M. Murray, Z. Li, and S. S. Sastry, A Mathematical Introduction to Robotic Manipulation. CRC, 1993.

[12] J. F. O'Brien, F. Jafari, and J. T. Wen, “Determination of unstable singularities in parallel robots with $\mathrm{N}$ arms," IEEE Transactions on Robotics, vol. 22, no. 1, pp. 160-167, 2006.

[13] B. Siciliano, L. Sciavicco, L. Villani, and G. Oriolo, Robotics: modelling, planning and control. Springer Verlag, 2009.

[14] J. T. Wen and J. F. O'Brien, "Singularities in threelegged platform-type parallel mechanisms," IEEE Transactions on Robotics and Automation, vol. 19, no. 4, 720-722 2003. 